\title{
Rational design for broadened substrate specificity and enhanced activity of a novel acetyl
}

xylan esterase from Bacteroides thetaiotaomicron

\section{Supporting Information}

Luyao Wang, ${ }^{\dagger, \nabla}$ Xue Han, $,{ }^{\dagger}, \nabla$, Yulu Wang, ${ }^{\dagger}$ Xue Wei, ${ }^{\dagger}$ Shujun Liu, ${ }^{\dagger}$ Shuli Shao, ${ }^{\ddagger}$, Shaoqing Yang,, Lichao Sun, ${ }^{*}, \uparrow$, and Fengjiao Xin ${ }^{*}, \uparrow$

${ }^{\dagger}$ Laboratory of Biomanufacturing and Food Engineering, Institute of Food Science and Technology, Chinese Academy of Agricultural Sciences, Beijing 100193, China

$¥$ College of Life Science and Agriculture Forestry, Qiqihar University, Qiqihar 161000, China

$\S$ Key Laboratory of Molecular Medicine and Biotherapy, School of Life Science, Beijing Institute of Technology, Beijing 100081, China

ฯ Key Laboratory of Food Bioengineering (China National Light Industry), College of Food Science and Nutritional Engineering, China Agricultural University, Beijing 100083, China

${ }^{\nabla}$ These authors contributed equally to this work.

Correspondence: Lichao Sun (Email: sun2004go@163.com, Tel/Fax: +86-10-62893472);

Fengjiao Xin (Email: 2002hongzhi30@163.com, Tel/Fax: 010-62815873)

This work was supported by National Key Research and Development Plan "modern food processing and food storage and transportation technology and equipment" (2017YFD0400204), National Natural Science Foundation of China (31700701 and 31801475), and Central Public-interest Scientific Institution Basal Research Fund (S2020JBKY-13). 


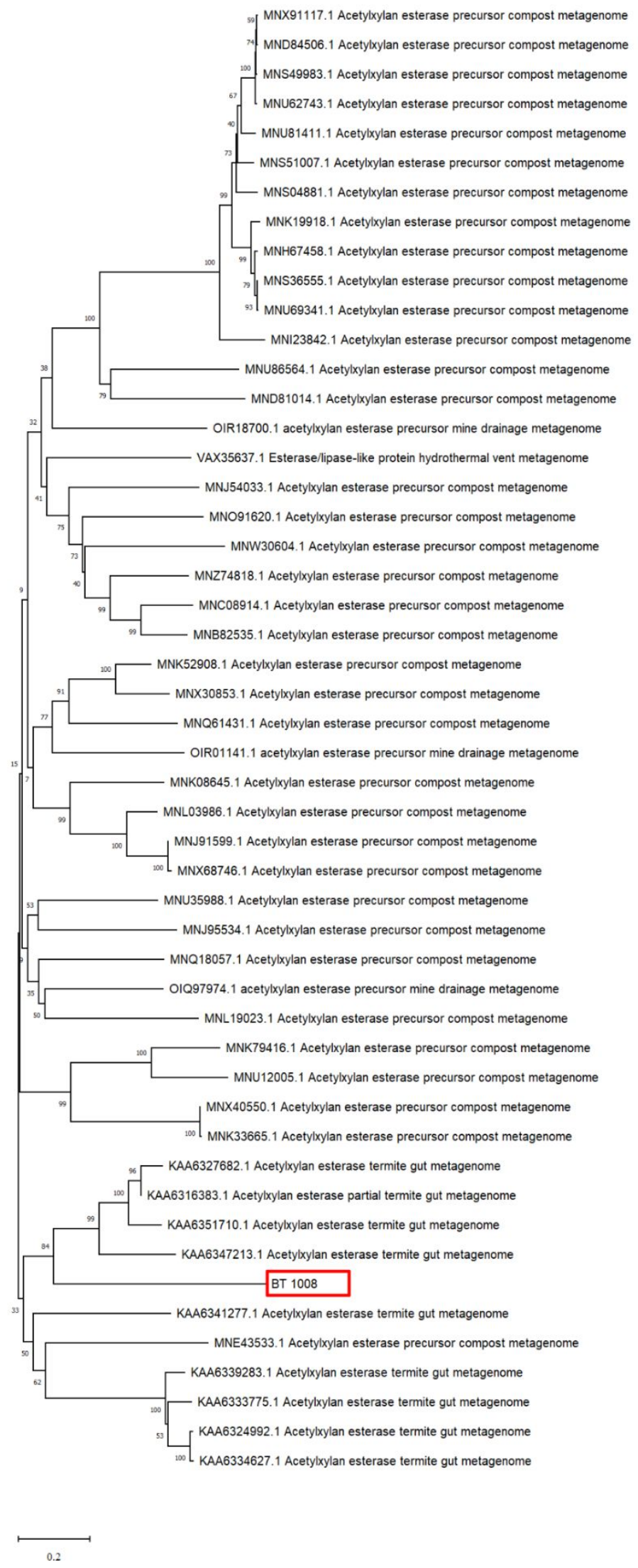

Figure S1. Sequence similarity phylogenetic tree of BTAxe1. The phylogenetic tree data comes from the NCBI metagenomic protein blast database. 

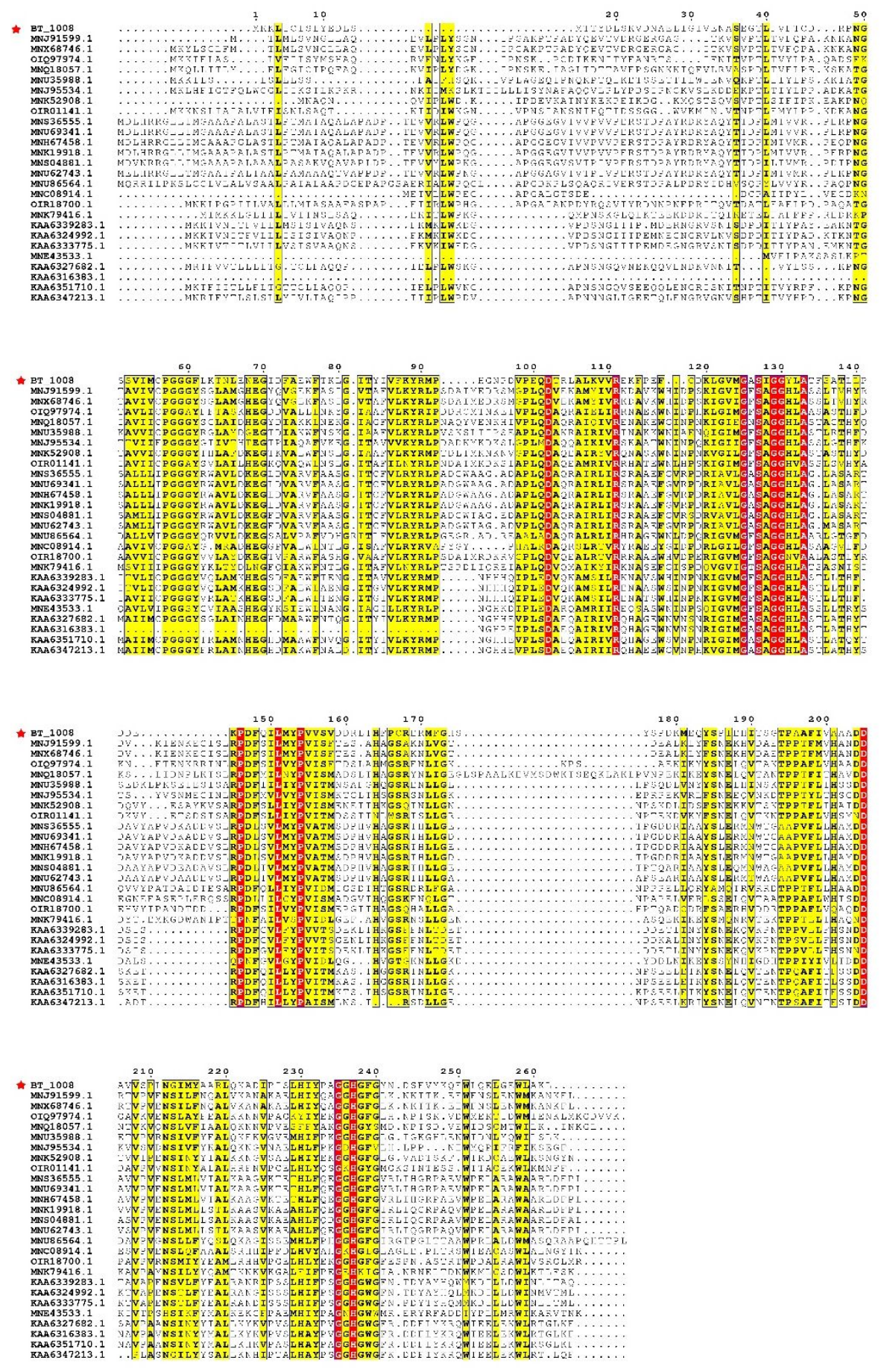

Figure S2. Amino acid sequence alignment of BTAxe1 with the referenced acetylxylan esterases. 


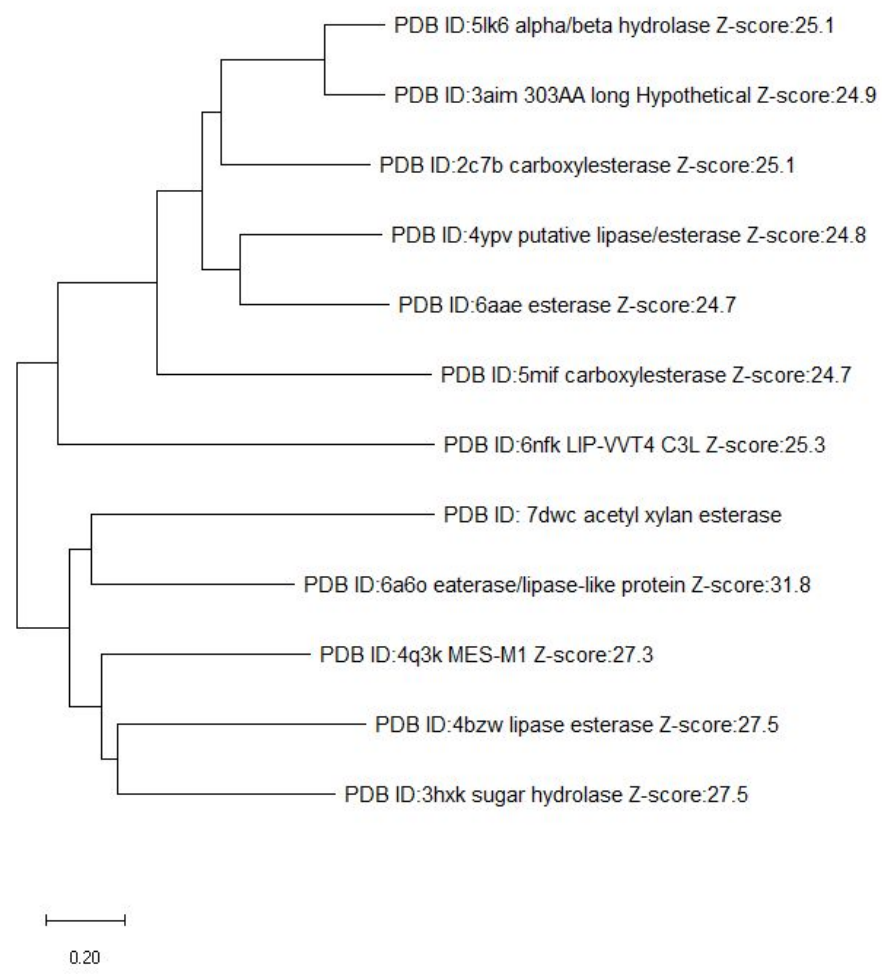

Figure S3. Structural similarity dendrogram of BTAxe1. The dendrogram was derived by average linkage clustering of the structural similarity matrix using the DALI server. PDB IDs were obtained from the Protein Data Bank. Zscores were obtained from the DALI server.

A

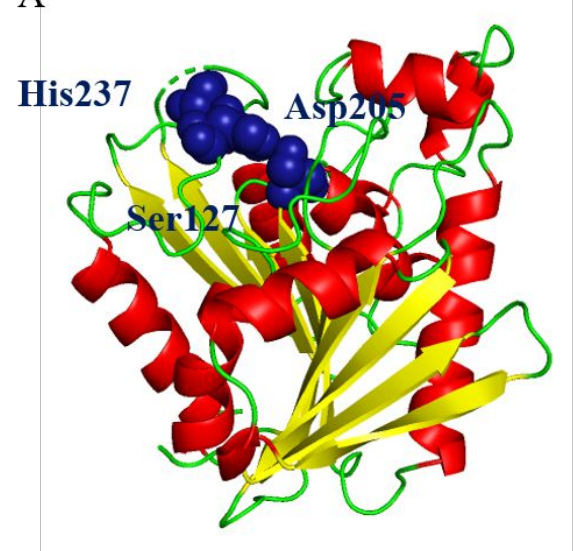

B

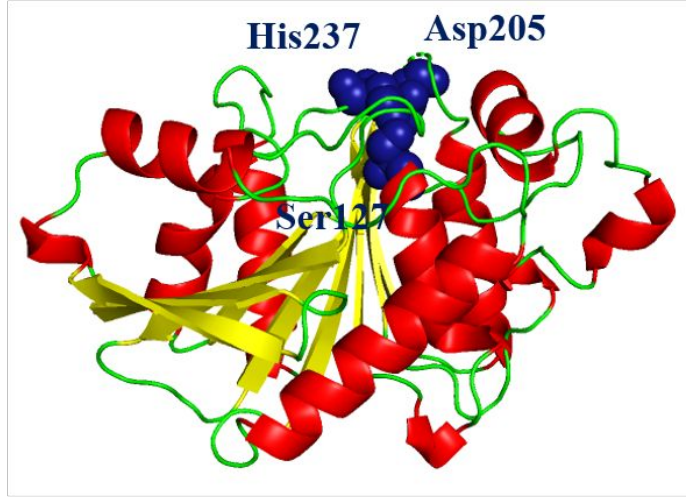

Figure S4. Three-dimensional protein structure diagram of BTAxe1 from different angles. 


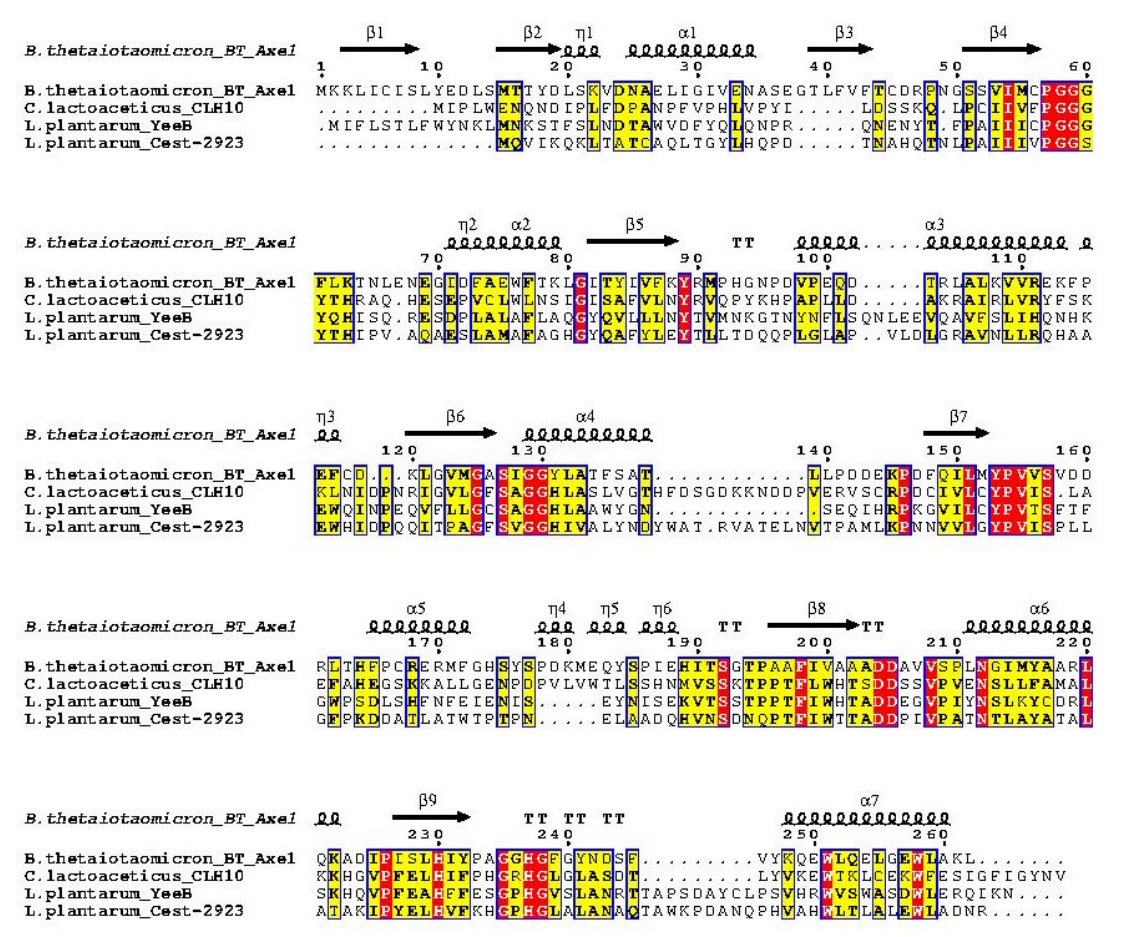

Figure S5. Amino acid sequence alignment of BTAxe1 with its closest homologs.

Table S1. Kinetic parameters of BTAxe1 and its putative acetyl ester active region mutants in the hydrolysis of $p$ NPA and $p$ NPB.

\begin{tabular}{|c|c|c|c|c|}
\hline Substrate & Enzyme & $K_{m}(\mathbf{m M})$ & $k_{c a t}\left(\min ^{-1}\right)$ & $k_{c a t} / K_{m}\left(\mathrm{mM}^{-1} \cdot \mathrm{min}^{-1}\right)$ \\
\hline \multirow{6}{*}{$p \mathrm{NPA}$} & Wild Type & $2.21 \pm 0.15$ & $26.88 \pm 2.82$ & $12.11 \pm 1.03$ \\
\hline & S127A & N.D. & N.D. & N.D. \\
\hline & D205A & N.D. & N. D. & N.D. \\
\hline & $\mathrm{H} 237 \mathrm{~A}$ & N.D. & N.D. & N.D. \\
\hline & G59A & $2.75 \pm 0.05$ & $1.20 \pm 0.16$ & $0.43 \pm 0.09$ \\
\hline & G60A & $2.35 \pm 0.08$ & $1.39 \pm 0.18$ & $0.59 \pm 0.07$ \\
\hline \multirow{6}{*}{$p$ NPB } & Wild Type & $4.57 \pm 0.34$ & $6.89 \pm 0.53$ & $1.51 \pm 0.24$ \\
\hline & S127A & N.D. & N.D. & N.D. \\
\hline & D205A & N.D. & N. D. & N.D. \\
\hline & $\mathrm{H} 237 \mathrm{~A}$ & N.D. & N.D. & N.D. \\
\hline & G59A & N.D. & N. D. & N.D. \\
\hline & G60A & N.D. & N.D. & N.D. \\
\hline
\end{tabular}

Note: N.D. not detectable. 
Table S2. Substrate specificity compared with BTAxe1 homologous proteins.

\begin{tabular}{cccc}
\hline & \multicolumn{3}{c}{$\boldsymbol{k}_{c a t} / \boldsymbol{K}_{\boldsymbol{m}}\left(\mathbf{m i n}^{-1} \mathbf{m M}^{-1}\right)$} \\
\cline { 2 - 4 } PDB ID & $\mathbf{C 2}(p \mathbf{N P A})$ & $\mathbf{C 4}(p \mathbf{N P B})$ & $\mathbf{C 8}(p \mathbf{N P O})$ \\
\hline 7DWC & 12.114 & 1.507 & Not detected \\
6A60 & $8.46 \times 10^{3}$ & Not found & Not found \\
4BZW & $1.12 \times 10^{4}$ & 162 & Not found \\
\hline
\end{tabular}

Note: N.D. not detectable. 